\title{
APPROXIMATION BY WEIGHTED MEANS OF WALSH-FOURIER SERIES
}

\author{
F. MÓRICZ* \\ Bolyai Institute, University of Szeged \\ Aradi vértanúk tere 1, 6720 Szeged, HUNGARY \\ B. E. RHOADES** \\ Department of Mathematics, Indiana University \\ Bloomington, Indiana 47405, U S A
}

(Received April 26, 1993 and in revised form February 20,1995)

\begin{abstract}
We study the rate of approximation to functions in $L^{p}$ and, in particular, in $\operatorname{Lip}(\alpha, p)$ by weighted means of their Walsh-Fourier series, where $\alpha>0$ and $1 \leq p \leq \infty$. For the case $p=\infty, L^{p}$ is interpreted to be $C_{W}$, the collection of uniformly $W$ continuous functions over the unit interval $[0,1)$. We also note that the weighted mean kernel is quasi-positive, under fairly general conditions.
\end{abstract}

1991. Mathematics Subject Classification. Primary 41A30.

KEY WORDS AND PHRASES: Walsh system, Walsh-Fourier series, weighted mean, rate of convergence, Lipschitz class, Walsh-Dirichlet kernel, Walsh-Fejér kernel, quasi-positive kernel.

\section{INTRODUCTION.}

We consider the Walsh orthonormal system $\left\{w_{k}(x): k \geq 0\right\}$ defined on the unit interval $I:=[0,1)$ using the Paley enumeration (see [4]).

Let $\mathcal{P}_{n}$ denote the collection of Walsh polynomials of order less than $n$; that is, functions of the form

$$
P(x):=\sum_{k=0}^{n-1} a_{k} w_{k}(x),
$$

where $n \geq 1$ and $\left\{a_{k}\right\}$ is any sequence of real numbers.

the approximation by Walsh polynomials in the norms of $L^{p}:=L^{p}(I), 1 \leq p<\infty$, and $C_{W}:=C_{W}(I)$. The class $C_{W}$ is the collection of all functions $f: I \rightarrow R$ that are uniformly continuous from the dyadic topology of $I$ into the usual topology of $R$; briefly, uniformly $W-$ continuous. The dyadic topology is generated by the collection of dyadic intervals of the form

$$
I_{m}:=\left[k 2^{-m},(k+1) 2^{-m}\right), \quad k=0,1, \ldots, 2^{m}-1 ; \quad m=0,1 \ldots
$$

For $C_{W}$ we shall write $L^{\infty}$. Set

$$
\begin{gathered}
\|f\|_{p}:=\left\{\int_{0}^{1}|f(x)|^{p} d x\right\}^{1 / p}, \quad 1 \leq p<\infty, \\
\|f\|_{\infty}:=\sup \{|f(x)|: x \in I\} .
\end{gathered}
$$

The best approximation of a function $f \in L^{p}, 1 \leq p \leq \infty$, by polynomials in $\mathcal{P}_{n}$ is defined by

$$
E_{n}\left(f, L^{p}\right):=\inf _{P \in \mathcal{P}_{n}}\|f-P\|_{p}
$$


For $f \in L^{p}$, the modulus of continuity is defined by

$$
\omega_{p}(f, \delta):=\sup \left\{\|f(\cdot \dot{+} t)-f(\cdot)\|_{p}:|t|<\delta\right\}
$$

where $\delta>0$ and $\dot{+}$ denotes dyadic addition. For $\alpha>0$, the Lipschitz classes in $L^{p}$ are defined by

$$
\operatorname{Lip}(\alpha, p):=\left\{f \in L^{p}: \omega_{p}(f, \delta)=\mathcal{O}\left(\delta^{\alpha}\right) \text { as } \delta \rightarrow 0\right\} .
$$

Concerning further properties and explanations, we refer the reader to [3], whose notations are adopted here as well.

\section{MAIN RESULTS.}

For $f \in L^{1}$, its Walsh-Fourier series is defined by

$$
\sum_{k=0}^{\infty} a_{k} w_{k}(x), \text { where } a_{k}:=\int_{0}^{1} f(t) w_{k}(t) d t .
$$

The $n$th partial sum of the series in $(2.1)$ is

$$
s_{n}(f, x):=\sum_{k=0}^{n-1} a_{k} w_{k}(x), \quad n \geq 1,
$$

which can also be written in the form

$$
s_{n}(f, x)=\int_{0}^{1} f(x \dot{+} t) D_{n}(t) d t
$$

where

$$
D_{n}(t):=\sum_{k=0}^{n-1} w_{k}(t), \quad n \geq 1,
$$

is the Walsh-Dirichlet kernel of order $n$.

Throughout, $\left\{p_{k}: k \geq 1\right\}$ will denote a sequence of nonnegative numbers, with $p_{1}>0$. The weighted means for series (2.1) are defined by

$$
t_{n}(f, x):=\frac{1}{P_{n}} \sum_{k=1}^{n} p_{k} s_{k}(f, x),
$$

where

$$
P_{n}:=\sum_{k=1}^{n} p_{k}, \quad n \geq 1
$$

We shall always assume that

$$
\lim _{n \rightarrow \infty} P_{n}=\infty
$$

which is the condition for regularity.

The representation

$$
t_{n}(f, x)=\int_{0}^{1} f(x \dot{+} t) L_{n}(t) d t
$$

plays a central role in the sequel, where

$$
L_{n}(t):=\frac{1}{P_{n}} \sum_{k=1}^{n} p_{k} D_{k}(t), \quad n \geq 1,
$$

is the weighted mean kernel. 
THEOREM 1. Let $f \in L^{p}, 1 \leq p \leq \infty, n:=2^{m}+k, 1 \leq k \leq 2^{m}, m \geq 1$.

(i) If $\left\{p_{k}\right\}$ is nondecreasing and satisfies the condition

$$
\frac{n p_{n}}{P_{n}}=\mathcal{O}(1)
$$

then

$$
\left\|t_{n}(f)-f\right\|_{p} \leq \frac{3}{P_{n}} \sum_{j=0}^{m-1} 2^{\jmath} p_{2^{\jmath+1}-1} \omega_{p}\left(f, 2^{-\jmath}\right)+\mathcal{O}\left(\omega_{p}\left(f, 2^{-m}\right)\right) .
$$

(ii) If $\left\{p_{k}\right\}$ is nonincreasing, then

$$
\left\|t_{n}(f)-f\right\|_{p} \leq \frac{3}{P_{n}} \sum_{j=0}^{m-1} 2^{j} p_{2}, \omega_{p}\left(f, 2^{-j}\right)+\mathcal{O}\left(\omega_{p}\left(f, 2^{-m}\right)\right) .
$$

THEOREM 2. Let $f \in \operatorname{Lip}(\alpha, p)$ for some $\alpha>0$ and $1 \leq p \leq \infty$. Then for $\left\{p_{k}\right\}$ nondecreasing,

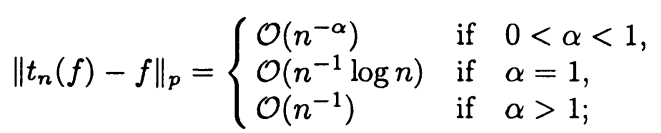

for $\left\{p_{k}\right\}$ nonincreasing,

$$
\left\|t_{n}(f)-f\right\|_{p}=\mathcal{O}\left(\frac{1}{P_{n}} \sum_{j=0}^{m-1} 2^{(1-\alpha) j} p_{2^{j}}+2^{-\alpha m}\right) .
$$

Given two sequences $\left\{p_{k}\right\}$ and $\left\{q_{k}\right\}$ of nonnegative numbers, we write $p_{k} \asymp q_{k}$ if there exist two positive constants $C_{1}$ and $C_{2}$ such that

$$
C_{1} q_{k} \leq p_{k} \leq C_{2} q_{k} \text { for all } k \text { large enough. }
$$

We present two particular cases for nonincreasing $\left\{p_{k}\right\}$.

Case (i): $p_{k} \asymp(\log k)^{-\beta}$ for some $\beta>0$. Then $P_{n} \asymp n(\log n)^{-\beta}$. It follows from (2.8)

$$
\left\|t_{n}(f)-f\right\|_{p}= \begin{cases}\mathcal{O}\left(n^{-\alpha}\right) & \text { if } 0<\alpha<1 \text { and } \beta>0 \\ \mathcal{O}\left(n^{-1} \log n\right) & \text { if } \alpha=1 \text { and } 0<\beta<1 \\ \mathcal{O}\left(n^{-1} \log n \log \log n\right) & \text { if } \alpha=\beta=1 \\ \mathcal{O}\left(n^{-1}(\log n)^{\beta}\right) & \text { if } \alpha=1 \text { and } \beta=1 \\ & \text { or if } \alpha>1 \text { and } \beta>0 .\end{cases}
$$

Case (ii): $p_{k} \asymp k^{-\beta}$ for some $0<\beta \leq 1$. Then $P_{n} \asymp n^{1-\beta}$ if $0<\beta<1$ and $P_{n} \asymp \log n$ if $\beta=1$. The case $\beta>1$ is unimportant since $P_{n}=\mathcal{O}(1)$. By (2.8),

$$
\left\|t_{n}(f)-f\right\|_{p}= \begin{cases}\mathcal{O}\left(n^{-\alpha}\right) & \text { if } \alpha+\beta<1 \\ \mathcal{O}\left(n^{\beta-1} \log n+n^{-\alpha}\right) & \text { if } \alpha+\beta=1 \\ \mathcal{O}\left(n^{\beta-1}\right) & \text { if } \alpha+\beta>1 \text { and } \beta>1 \\ \mathcal{O}\left((\log n)^{-1}\right) & \text { if } \beta=1,\end{cases}
$$

where $\alpha>0$ and $\beta>0$.

REMARK 1. The slower $P_{n}$ tends to infinity, the worse is the rate of approximation.

REMARK 2. Watari [6] has shown that a function $f \in L^{p}$ belongs to $\operatorname{Lip}(\alpha, p)$ for some $\alpha>0$ and $1 \leq p \leq \infty$ if and only if

$$
E_{n}\left(f, L^{p}\right)=\mathcal{O}\left(n^{-\alpha}\right)
$$


Thus, for $0<\alpha<1$, the rate of approximation to functions $f$ in the class $\operatorname{Lip}(\alpha, p)$ by $t_{n}(f)$ is as good as the best approximation.

REMARK 3. For $\alpha>1$, the rate of approximation by $t_{n}(f)$ in the class $\operatorname{Lip}(\alpha, p)$ cannot be improved too much.

THEOREM 3. If for some $f \in L^{p}, 1 \leq p \leq \infty$,

$$
\left\|t_{2^{m}}(f)-f\right\|_{p}=o\left(P_{2^{m}}^{-1}\right) \text { as } m \rightarrow \infty
$$

then $f$ is a constant.

If $p_{k}=1$ for all $k$, then the $t_{n}(f, x)$ are the $(C, 1)$ - means (i.e., the first arithmetic means) of the series in (2.1). In this case, Theorem 2 was proved by Yano [8] for $0<\alpha<1$ and by Jastrebova [1] for $\alpha=1$; Theorem 3 also reduces to a known result (see e.g. [5, p. 191]).

\section{AUXILIARY RESULTS}

Let

$$
K_{n}(t):=\frac{1}{n} \sum_{k=1}^{n} D_{k}(t), \quad n \geq 1
$$

by the Walsh-Fejer kernel.

LEMMA 1. (see [7]). Let $m \geq 0$ and $n \geq 1$. Then $K_{2^{m}}(t) \geq 0$ for each $t \in I$,

$$
\int_{0}^{1}\left|K_{n}(t)\right| d t \leq 2, \quad \text { and } \quad \int_{0}^{1} K_{2^{m}}(t) d t=1 .
$$

LEMMA 2. Let $n:=2^{m}+k, 1 \leq k \leq 2^{m}$, and $m \geq 1$. Then for $L_{n}(t)$ defined in (2.3),

$$
\begin{aligned}
P_{n} L_{n}(t)= & -\sum_{j=0}^{m-1} r_{j}(t) w_{2^{j}-1}(t) \sum_{i=1}^{2^{j}-1} i\left(p_{2^{j+1}-i}-p_{2^{j+1}-i-1}\right) K_{i}(t) \\
& -\sum_{j=0}^{m-1} r_{j}(t) w_{2^{j}-1}(t) 2^{j} p_{2^{j}} K_{2^{j}}(t) \\
& +\sum_{j=0}^{m-1}\left(P_{2^{j+1}-1}-P_{2^{j}-1}\right) D_{2^{j+1}}(t) \\
& +\left(P_{n}-P_{n-k-1}\right) D_{2^{m}}(t)+r_{m}(t) \sum_{i=1}^{k} p_{2^{m}+i} D_{i}(t),
\end{aligned}
$$

where the $r_{j}(t)$ are the Rademacher functions.

Proof. From (2.3)

$$
\begin{aligned}
P_{n} L_{n}(t) & =\sum_{i=1}^{2^{m}-1} p_{i} D_{i}(t)+\sum_{i=2^{m}}^{2^{m}+k} p_{i} D_{i}(t) \\
& =\sum_{j=0}^{m-1} \sum_{i=0}^{2^{j}-1} p_{2^{j}+i} D_{2^{j}+i}(t)+\sum_{i=0}^{k} p_{2^{m}+i} D_{2^{m}+i}(t) \\
& =\sum_{j=0}^{m-1} \sum_{i=0}^{2^{j}-1} p_{2^{j}+i}\left(D_{2^{j}+i}(t)-D_{2^{j}+1}(t)\right) \\
& +\sum_{j=0}^{m-1} D_{2^{j}+1}(t) \sum_{i=0}^{2^{j}-1} p_{2^{j}+i}+\sum_{i=0}^{k} p_{2^{m}+i} D_{2^{m}+i}(t)
\end{aligned}
$$

We will make use of formula (3.4) of [3]: 


$$
D_{2^{\jmath+1}}(t)-D_{2^{\jmath}+i}(t)=r_{\jmath}(t) w_{2^{j-1}}(t) D_{2_{-\imath}}(t), \quad 0 \leq i<2^{j},
$$

and the formula in line 4 from below of $[4, p .46]$ :

$$
D_{2^{m}+i}(t)=D_{2^{m}}(t)+r_{m} D_{i}(t), \quad 1 \leq i \leq 2^{m} .
$$

Substituting these into (3.3) yields

$$
\begin{aligned}
P_{n} L_{n}(t)= & -\sum_{j=0}^{m-1} r_{j}(t) w_{2^{j}-1}(t) \sum_{i=0}^{2^{J}-1} p_{2^{\jmath}+{ }_{\imath}} D_{2^{j}-\imath}(t) \\
& +\sum_{\imath=0}^{m-1}\left(P_{2^{j+\imath}}-P_{2,-1}\right) D_{2 J_{-i}}(t) \\
& +\left(P_{n}-P_{n-k-1}\right) D_{2^{m}}(t)+r_{m}(t) \sum_{i=1}^{k} p_{2^{m}+\imath} D_{\imath}(t) .
\end{aligned}
$$

Hence (3.2) follows, by noting that

$$
D_{i}(t)=i K_{i}(t)-(i-1) K_{i-1}(t), \quad i \geq 1, \quad K_{0}(t):=0,
$$

(see (3.1)) and accordingly

$$
\begin{gathered}
\sum_{i=0}^{2^{j}-1} p_{2^{j}+i} D_{2^{\jmath}-i}(t)=\sum_{i=1}^{2^{j}} p_{2^{j+1}-i} D_{\imath}(t) \\
=\sum_{i=1}^{2^{j}-1} i\left(p_{2^{j+1-\imath}}-p_{2^{j+1}-i-1}\right) K_{i}(t)+2^{j} p_{2^{j}} K_{2}(t) .
\end{gathered}
$$

Motivated by (3.2), we define a linear operator $R_{n}$ by

$$
R_{n}(t):=\frac{1}{P_{n}} \sum_{i=1}^{k} p_{2^{m}+i} D_{2}(t),
$$

where $n:=2^{m}+k, 1 \leq k \leq 2^{m}$, and $m \geq 1$. A Sidon type inequality of [2] implies that $R_{n}$ as well as the weighted mean kernel $L_{n}$ defined in (2.3) are quasi-positive.

LEMMA 3. Let $\left\{p_{k}\right\}$ be a sequence of nonnegative numbers either nondecreasing and satisfying condition (2.4) or merely nonincreasing, and let $R_{n}$ be defined by (3.4). Then there exists a constant $C$ such that

$$
I_{n}:=\int_{0}^{1}\left|R_{n}(t)\right| d t \leq C, \quad n \geq 1 .
$$

PROOF. By [2, Lemma 1 for $p=2]$,

$$
I_{n} \leq \frac{4 k^{1 / 2}}{P_{n}}\left(\sum_{i=1}^{k} p_{2^{m}+i}^{2}\right)^{1 / 2}
$$

Due to monotonicity,

$$
I_{n} \leq \begin{cases}\frac{4 k p_{n}}{P_{n}} \leq \frac{2 n p_{n}}{P_{n}} & \text { if }\left\{p_{k}\right\} \text { is nondecreasing, } \\ \frac{4 k p_{2} m_{+1}}{P_{n}} \leq 4 & \text { if }\left\{p_{k}\right\} \text { is nonincreasing. }\end{cases}
$$

By (2.4), hence (3.5) follows. 
LEMMA 4 (see [3]). If $g \in \mathcal{P}_{2^{m}}, f \in L^{p}$, where $m \geq 0$ and $1 \leq p \leq \infty$, then

$$
\left\|\int_{0}^{1} r_{m}(t) g(t)[f(\cdot \dot{+} t)-f(\cdot)] d t\right\|_{p} \leq 2^{-1} \omega_{p}\left(f, 2^{-m}\right)\|g\|_{1}
$$

4. PROOFS OF THEOREMS 1-3.

PROOF OF THEOREM 1. We shall present the details only for $1 \leq p<\infty$. By (2.2), (3.2), and the usual Minkowski inequality,

$$
\begin{aligned}
P_{n}\left\|t_{n}(f)-f\right\|_{p} & =\left\{\int_{0}^{1}\left|\int_{0}^{1} P_{n} L_{n}(t)[f(x+t)-f(x)] d t\right|^{p} d x\right\}^{1 / p} \\
& \leq \sum_{j=0}^{m-1}\left\{\int_{0}^{1}\left|\int_{0}^{1} r_{j}(t) g_{j}(t)[f(x \dot{+} t)-f(x)] d t\right|^{p} d x\right\}^{1 / p} \\
& +\sum_{j=0}^{m-1}\left\{\int_{0}^{1}\left|\int_{0}^{1} r_{j}(t) h_{j}(t)[f(x+t)-f(x)] d t\right|^{p} d x\right\}^{1 / p} \\
& +\sum_{j=0}^{m-1}\left(P_{2^{j+1}-1}-P_{2^{j}-1}\right)\left\{\int_{0}^{1}\left|\int_{0}^{1} D_{2^{j+1}}(t)[f(x \dot{+} t)-f(x)] d t\right|^{p} d x\right\}^{1 / p} \\
& +\left(P_{n}-P_{n-k-1}\right)\left\{\int_{0}^{1}\left|\int_{0}^{1} D_{2^{m}}(t)[f(x+t)-f(x)] d t\right|^{p} d x\right\}^{1 / p} \\
& +P_{n}\left\{\int_{0}^{1}\left|\int_{0}^{1} r_{m}(t) R_{n}(t)[f(x+t)-f(x)] d t\right|^{p} d x\right\}^{1 / p} \\
& =: I_{1 n}+I_{2 n}+I_{3 n}+I_{4 n}+I_{5 n}, \quad \text { say, }
\end{aligned}
$$

where

From Lemma 1,

$$
\begin{gathered}
g_{j}(t):=w_{2^{j}-1}(t) \sum_{i=1}^{2^{j}-1} i\left(p_{2^{j+1}-i}-p_{2^{j+1}-i-1}\right) K_{i}(t) \\
h_{j}(t):=2^{j} p_{2^{j}} w_{2^{j-1}}(t) K_{2^{j}}(t) .
\end{gathered}
$$

$$
\begin{aligned}
\int_{0}^{1}\left|g_{j}(t)\right| d t & \leq \sum_{i=1}^{2^{j}-1} i\left|p_{2^{j+1}-i}-p_{2^{j+1}-i-1}\right| \int_{0}^{1}\left|K_{i}(t)\right| d t \\
& \leq 2 \sum_{r=2^{j+1}}^{2^{j+1}-1}\left(2^{j+1}-r\right)\left|p_{r}-p_{r-1}\right|=: A_{j}, \quad \text { say }
\end{aligned}
$$

If $\left\{p_{k}\right\}$ is nondecreasing, we have

$$
\begin{aligned}
A_{j} & =2^{j+2} \sum_{r=2^{j+1}}^{2^{j+1}-1}\left(p_{r}-p_{r-1}\right)-2 \sum_{r=2^{j}+1}^{2^{j+1}-1}\left(r p_{r}-(r-1) p_{r-1}\right)+2 \sum_{r=2^{j+1}}^{2^{j+1}-1} p_{r-1} \\
& =2^{j+2}\left(p_{2^{j+1}-1}-p_{2^{j}}\right)-2\left[\left(2^{j+1}-1\right) p_{2^{j+1}-1}-2^{j} p_{2^{j}}\right]+2\left(P_{2^{j+1}-2}-P_{2^{j}-1}\right) \\
& <2\left(P_{2^{j+1}-1}-P_{2^{j}-1}\right) \leq 2^{j+1} p_{2^{j+1}-1} .
\end{aligned}
$$

If $\left\{p_{k}\right\}$ is nonincreasing, we have

$$
A_{j}=2^{j+2} \sum_{r=2^{j}+1}^{2^{j+1}-1}\left(p_{r-1}-p_{r}\right)+2 \sum_{r=2^{j}+1}^{2^{j+1}-1}\left(r p_{r}-(r-1) p_{r-1}\right)-2 \sum_{r=2^{j}+1}^{2^{j+1}-1} p_{r-1}<2^{j+1} p_{2^{j}}
$$


Thus, by Lemma 4 , for $\left\{p_{k}\right\}$ nondecreasing,

$$
I_{1 n} \leq \sum_{j=0}^{m-1} 2^{j} p_{2 j+1-1} \omega_{p}\left(f, 2^{-j}\right),
$$

and for $\left\{p_{k}\right\}$ nonincreasing,

$$
I_{1 n} \leq \sum_{j=0}^{m-1} 2^{j} p_{2^{j}} \omega_{p}\left(f, 2^{-j}\right) .
$$

Again, by Lemmas 1 and 4,

$$
\int_{0}^{1}\left|h_{j}(t)\right| d t \leq 2^{j} p_{2^{j}} \int_{0}^{1} K_{2^{j}}(t) d t=2^{j} p_{2^{j}}
$$

whence

$$
I_{2 n} \leq 2^{-1} \sum_{j=0}^{m-1} 2^{j} p_{2^{j}} \omega_{p}\left(f, 2^{-j}\right)
$$

Since

$$
D_{2^{m}}(t)= \begin{cases}2^{m} & \text { if } t \in\left[0,2^{-m}\right), \\ 0 & \text { if } t \in\left[2^{-m}, 1\right)\end{cases}
$$

(see, e.g., [5, p.7]), by the generalized Minkowski inequality,

$$
\begin{aligned}
I_{3 n} & \leq \sum_{j=0}^{m-1}\left(P_{2^{j+1}-1}-P_{2^{j}-1}\right) \int_{0}^{1} D_{2^{j+1}}(t)\left\{\int_{0}^{1}|f(x+t)-f(x)|^{p} d x\right\}^{1 / p} d t \\
& \leq \sum_{j=0}^{m-1}\left(P_{2^{j+1}-1}-P_{2^{j}-1}\right) \omega_{p}\left(f, 2^{-j-1}\right)
\end{aligned}
$$

and

$$
I_{4 n} \leq\left(P_{n}-P_{n-k-1}\right) \omega_{p}\left(f, 2^{-m}\right)
$$

Note that

$$
P_{2^{j+1}-1}-P_{2^{j-1}} \leq \begin{cases}2^{j} p_{2^{j+1}-1} & \text { if }\left\{p_{k}\right\} \text { is nondecreasing, } \\ 2^{j} p_{2^{j}} & \text { if }\left\{p_{k}\right\} \text { is nonincreasing. }\end{cases}
$$

By Lemmas 3 and 4 ,

$$
\begin{aligned}
I_{5 n} & \leq 2^{-1} P_{n} \omega_{p}\left(f, 2^{-m}\right) \int_{0}^{1}\left|R_{n}(t)\right| d t \\
& \leq 2^{-1} C P_{n} \omega_{p}\left(f, 2^{-m}\right) .
\end{aligned}
$$

Combining (4.1) - (4.8) yields (2.5) and (2.6).

PROOF OF THEOREM 2. For $\left\{p_{k}\right\}$ nondecreasing we have, from (2.4) and (2.5),

$$
\begin{aligned}
\left\|t_{n}(f)-f\right\|_{p} & =\mathcal{O}\left(\frac{p_{2 m}}{P_{n}} \sum_{j=0}^{m-1} 2^{(1-\alpha) j}+2^{-\alpha m}\right) \\
& =\mathcal{O}\left(2^{-m} \sum_{j=0}^{m-1} 2^{(1-\alpha) j}+2^{-\alpha m}\right) .
\end{aligned}
$$

Hence (2.7) follows easily. 
For $\left\{p_{k}\right\}$ nonincreasing, $(2.8)$ is immediate.

PROOF OF THEOREM 3. By a theorem of Watari [6]

$$
\left\|s_{2^{m}}(f)-f\right\|_{p} \leq 2 E_{2^{m}}\left(f, L^{p}\right) .
$$

Thus, from (2.9),

$$
\left\|s_{2^{m}}(f)-f\right\|_{p}=o\left(P_{2^{m}}^{-1}\right)
$$

Clearly,

$$
\begin{aligned}
P_{2^{m}}\left\{s_{2^{m}}(f, x)\right. & \left.-t_{2^{m}}(f, x)\right\}=\sum_{k=1}^{2^{m}} p_{k}\left\{s_{2^{m}}(f, x)-s_{k}(f, x)\right\} \\
& =\sum_{k=1}^{2^{m}-1} p_{k} \sum_{i=k}^{2^{m}-1} a_{\imath} w_{\imath}(x) \\
& =\sum_{i=1}^{2^{m}-1} P_{\imath} a_{\imath} w_{i}(x) .
\end{aligned}
$$

Now (2.9) and (4.9) imply

$$
\lim _{m \rightarrow \infty}\left\|\sum_{i=1}^{2^{m}-1} P_{i} a_{i} w_{i}(x)\right\|_{p}=0 .
$$

Since the $L^{p}$-norm dominates the $L^{1}$-norm for $p>1$, it follows that for $j \geq 1$,

$$
\begin{aligned}
\left|P_{j} a_{\jmath}\right| & =\lim _{m \rightarrow \infty}\left|\int_{0}^{1} w_{j}(x) \sum_{i=1}^{2^{m}-1} P_{i} a_{i} w_{i}(x) d x\right| \\
& \leq \lim _{m \rightarrow \infty}\left\|\sum_{i=1}^{2^{m}-1} P_{i} a_{i} w_{i}(x)\right\|_{1}=0 .
\end{aligned}
$$

Hence we conclude that $a_{j}=0$ for all $j \geq 1$. Therefore, $f=a_{0}$, a constant.

* This research was partially supported by the Hungarian National Foundation for Scientific Research under Grant\#234.

** This research was completed while the author was a Fulbright scholar at the Bolyai Institute, University of Szeged, Hungary, during the fall semester in the academic year 1992/93.

\section{REFERENCES}

1. JASTREBOVA, M.A., On approximation of functions satisfying the Lipschitz condition by arithmetic means of their Walsh-Fourier series, Mat. Sb. 71 (1966), 214-226.

2. MÓRICZ, F. and SCHIPP, F., On the integrability and $L^{1}$-convergence of Walsh series with coefficients of bounded variation, J. Math. Anal. Appl. 146 (1990), 99-109.

3. MÓRICZ, F. and SIDDIQI, A.H., Approximation by Nörlund means of Walsh-Fourier series, J. Approx. Theory 70 (1992), 375-389.

4. PALEY, R.E.A.C., A remarkable system of orthogonal functions, Proc. London, Math. Soc. 34 (1932), 241-279.

5. SCHIPP, F., WADE, W.R., and SIMON, P., "Walsh Series. An introduction to Dyadic Harmonic Analysis", Adadémiai Kiadó, Budapest, 1990.

6. WATARI, C., Best approximation by Walsh polynomials, Tôhoku Math. J. 15 (1963), 1-5.

7. YANO, SH., On Walsh series, Tôhoku Math. J. 3 (1951), 223-242.

8. YANO, SH., On approximation by Walsh functions, Proc. Amer. Math. Soc. 2 (1951), 962-967. 


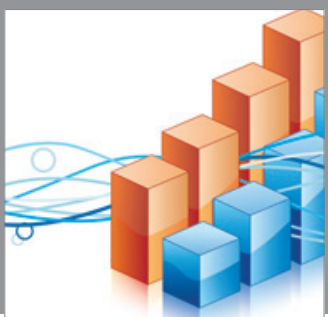

Advances in

Operations Research

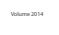

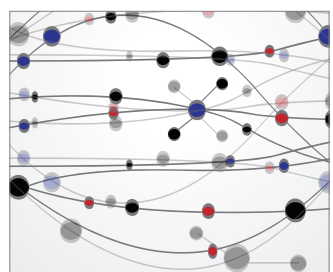

\section{The Scientific} World Journal
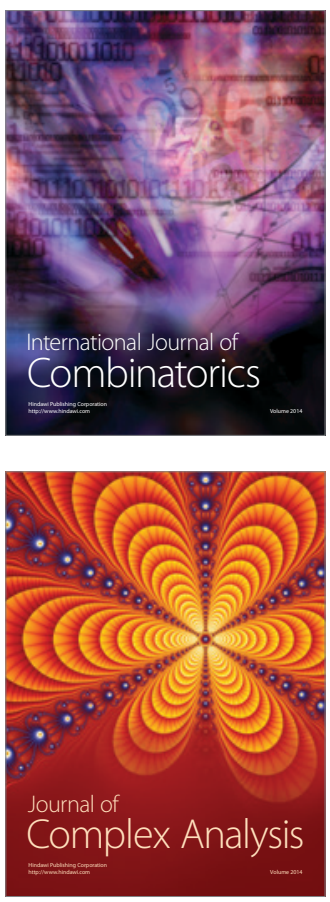

International Journal of

Mathematics and

Mathematical

Sciences
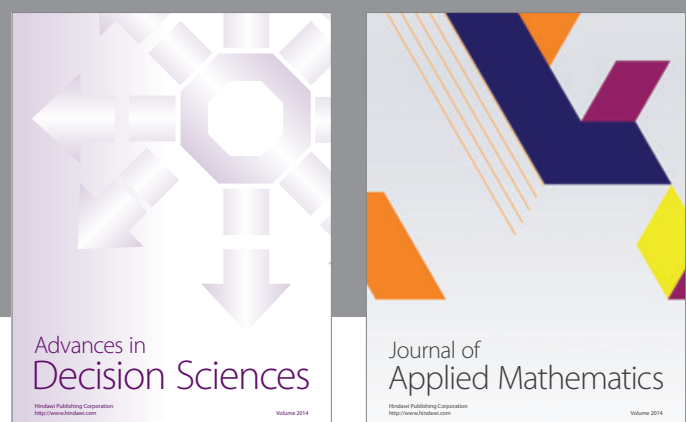

Journal of

Applied Mathematics
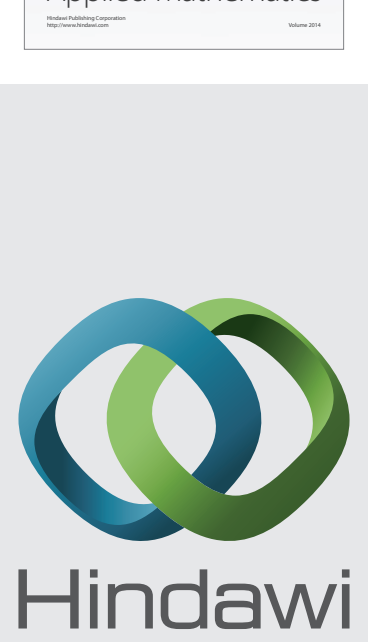

Submit your manuscripts at http://www.hindawi.com
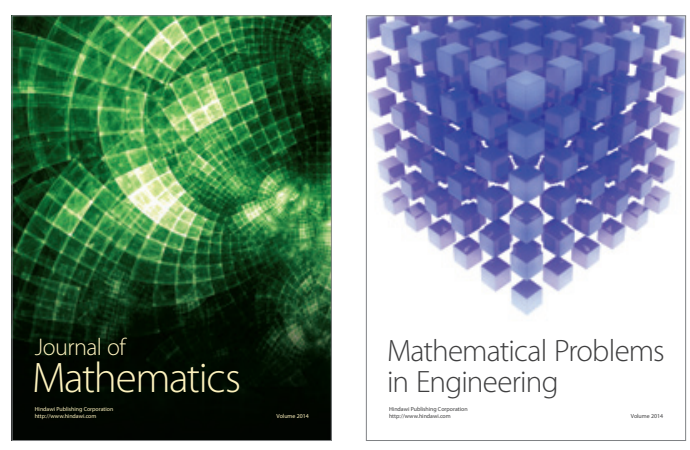

Mathematical Problems in Engineering
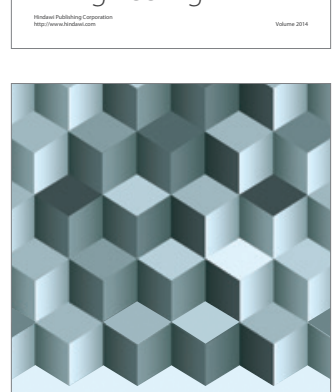

Journal of

Function Spaces
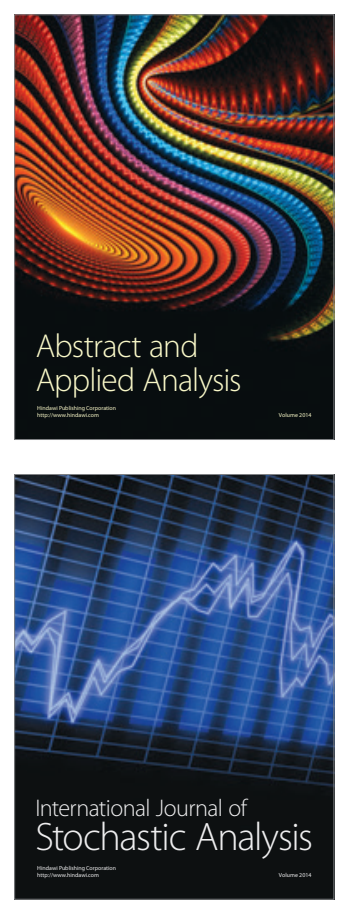

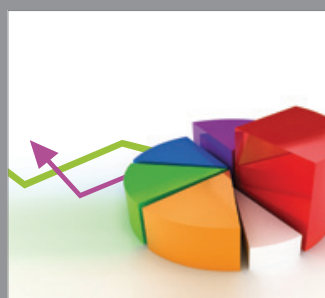

ournal of

Probability and Statistics

Promensencen
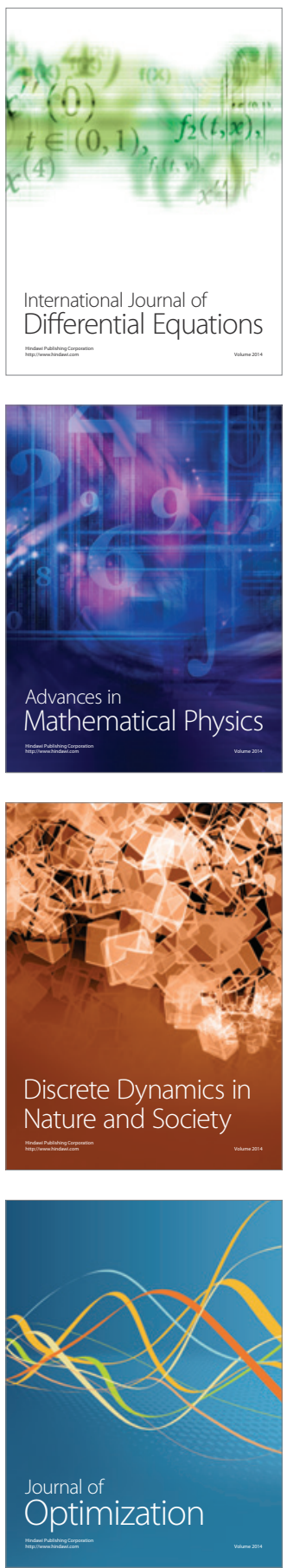